\title{
The evolution of viral emergence
}

\author{
Edward C. Holmes* \\ Center for Infectious Disease Dynamics, Department of Biology, Pennsylvania State University, University Park, PA 16802
}

$\mathrm{T}$ he current uncertainty over whether H5N1 avian influenza virus will successfully adapt to human transmission highlights the importance of understanding the evolutionary basis of viral emergence, particularly the respective roles played by ecology and genetics in allowing viruses to establish productive transmission networks in new host species. Work by Anishchenko et al. (1) in this issue of PNAS reveals that even small changes in viral genomes can have a fundamental impact on fitness in new hosts, therein facilitating disease emergence.

Although RNA viruses are often able to adapt to changing environments with remarkable rapidity, an adaptability that stems from their high mutation rates and large population sizes (2), it is also the case that the evolution of ongoing transmission in new host species is rather harder to achieve. Indeed, of the many RNA viruses that are categorized as "emerging," the great majority is in reality "spillover" infections with little or no subsequent transmission. The transmission of avian $\mathrm{H} 5 \mathrm{~N} 1$ virus in humans is a perfect case in point; despite numerous avian-to-human spillover infections, to date there is no compelling evidence of secondary, sustained human-to-human transmission, the crucial step in establishing a full-blown epidemic. This pattern of repeated exposure without large-scale transmission seems particularly typical of arthropodborne RNA viruses ("arboviruses"), which are a common cause of spillover infections but rarely fully adapt to new host transmission cycles. The answer to this apparent paradox (why rapidly evolving RNA viruses often have trouble in achieving what might appear to be simple adaptive solutions) is that adaptation to new hosts is often polygenic involving multiple mutations in a variety of genes and which may exhibit complex fitness tradeoffs. In population genetic terms, a virus has to move from one fitness peak (successful transmission in the reservoir host species) to another (successful transmission in the recipient host species) by traversing a valley of low fitness where the virus is inadequately adapted to both species. In the absence of high rates of recombination or reassortment, such large jumps in fitness are never easy to achieve because they may require the simultaneous occurrence of multiple advantageous mutations in a single replication cycle.
In some cases, however, far fewer mutational changes seem to be required to enable a species jump, so that fitness valleys might be more accurately depicted as very narrow ravines. This limited genetic change is the key observation made by Anishchenko et al. (1). The subject of study in this case was Venezuelan equine encephalitis virus (VEEV), a mosquito-borne RNA virus that on occasion emerges from its enzootic cycle involving rodents and an array of mosquito species to cause serious epidemics of neurological disease in horses (with humans as a spillover host; see ref. 3). Anishchenko et al. show that a single amino acid change in the E2 envelope glycoprotein Thr-213 $\rightarrow$ Lys (Thr to Lys at position 213) is sufficient to explain how an enzootic and equine avirulent VEEV strain (serotype 1D)

\section{Ecological and immunological, rather than genetic, factors are the main determinant of successful emergence.}

was able to adapt to horses and cause a small-scale epidemic among both equines and humans during 1992-1993 (serotype 1C). Such an observation is important not just for understanding the evolution of emergence in VEEV, but also because it represents one of the very few cases in which the genetics of host adaptation is known for any virus. To put the novelty of this result in context, although HIV-1 was first isolated in 1983, the mutations required for its successful emergence in humans after cross-species transmission from its chimpanzee reservoir are still unknown.

\section{Detecting Adaptive Mutations}

An equally noteworthy aspect of the Anishchenko et al. (1) study is the fertile marriage of comparative and experimental studies of viral evolution. Although the genome sequences of RNA viruses often show great genetic variability, a simple evolutionary technique was applied to sieve through this potentially bewildering mass of diversity; the amino acid changes chosen for further study were those that fell on the branch leading to the VEEV strains associated with the 1992-1993 epidemic and, hence, that define this outbreak strain compared with its enzootic ancestor. In the crucial E2 protein, such a survey recovered just two amino acid changes, the aforementioned Thr-213 $\rightarrow$ Lys as well as Gly-193 $\rightarrow$ Arg, both of which are located in an immunodominant region. Strikingly, a prior phylogeny-based analysis of selection pressures in VEEV had also pinpointed site 213 as the target of positive selection (adaptive evolution) in that it was subject to a particularly high rate of amino acid change compared with the background rate of synonymous mutation (4). Positive selection might be expected to follow viral emergence in a new host species because this event would obviously entail a major boost in the number of susceptible hosts and a concomitant increase in fitness. To test whether the phylogenetic prediction was correct, Anishchenko et al. used reverse genetic techniques to introduce the Thr$213 \rightarrow$ Lys and Gly-193 $\rightarrow$ Arg mutations, singly and in combination, into a VEEV infectious clone and then measured fitness both in vitro and in vivo. Both sets of experiments pointed to Thr-213 $\rightarrow$ Lys alone as the most likely suspect for the equine emergence of VEEV, and this mutation was also able to duplicate the $1 \mathrm{D}$ to $1 \mathrm{C}$ serotypic change. Particularly noteworthy was that, in vivo, the single introduction of Thr-213 $\rightarrow$ Lys was sufficient to produce both the clinical symptoms and high viremia comparable with that observed in true equine VEEV epidemics. The only uncertain element in this seemingly open-and-shut case is the function, if any, of the Gly-193 $\rightarrow$ Arg mutation. Given that this latter change appears to have little effect on fitness (or disease), either beneficial or detrimental, it may simply represent a neutral amino acid change that has swept to fixation because of physical linkage to the beneficial amino acid replacement at site 213 .

\section{Ecology and Genetics in Emergence}

The conclusion that Anishchenko et al. (1) draw from their study is that, in the case of VEEV, ecological and immuno-

Conflict of interest statement: No conflicts declared. See companion article on page 4994.

*E-mail: ech15@psu.edu.

๑ 2006 by The National Academy of Sciences of the USA 
logical, rather than genetic, factors are the main determinant of successful emergence, with epizootics in horses strongly associated with an abundance of floodwater mosquitoes in marsh or pasture habitats. Such a conclusion might be more broadly applicable too, because it is clear that a key parameter in initiating the process of viral emergence is the proximity and density of donor and recipient host species; the more contact between two species, and the larger and more dense their populations, then the more likely that they will exchange pathogens. In this sense, it is no surprise that with recent changes in human ecology, most notably increases in population size and urbanization, deforestation, and ever greater global mobility, so our burden of infectious diseases has similarly increased and will evidently continue to do so. However, VEEV may also be unusual in requiring so few mutations to facilitate emergence. In particular, because VEEV periodically emerges in horses from its

1. Anishchenko, M., Bowen, R. A., Paessler, S., Austgen, L., Greene, I. P. \& Weaver, S. C. (2006) Proc. Natl. Acad. Sci. USA 103, 4994-4999.

2. Domingo, E. \& Holland, J. J. (1997) Annu. Rev. Microbiol. 51, 151-178.

3. Walton, T. E. \& Grayson, M. A. (1988) in Epide- enzootic background, it must be the case that this virus already possesses nearly all of the genetic factors necessary to allow sustained transmission in horses. Similarly, the Thr-213 $\rightarrow$ Lys mutation seemed to have little effect on fitness in the Aedes taeniorhynchus mosquito vector associated with VEEV transmission in horses (5), whereas far greater fitness tradeoffs are seen in other mammalmosquito transmission systems (6). Hence, successful viral emergence in a completely novel host with no previous history of transmission is likely to represent a far larger adaptive challenge. For example, in the case of the carnivore parvoviruses, where the genetics of adaptation have also been explored in detail, a handful of amino acid changes were necessary for the virus to successfully jump from cats to dogs in the early 1970s (7). The current failure of H5N1 to establish human-to-human transmission suggests that multiple mutations are also required in the case of influenza virus, although to be complacent about

miology and Ecology, The Arboviruses, ed. Monath, T. P. (CRC, Boca Raton, FL), Vol. IV, pp. 203-231.

4. Brault, A. C., Powers, A. M., Holmes, E. C., Woelk, C. H. \& Weaver, S. C. (2002) J. Virol. 76, 1718-1730.

5. Ortiz, D. I. \& Weaver, S. C. (2004) J. Med. the likelihood of further evolution would be foolhardy.

The study of Anishchenko et al. (1) points to a new synthesis, and one that is essential for the study of viral emergence to truly come of age. Specifically, it will be necessary to meld into one unified framework experimental studies on the molecular basis of viral adaptation to new hosts, both in vitro and where possible in vivo, the phylogenetic analysis of viral sequences derived from donor and recipient host species and that provide a crucial insight into evolution in nature, and epidemiological studies on the fundamental mechanisms and dynamics of transmission. The current study represents a valuable forward step in this synthesis because it elegantly shows how a combination of comparative and experimental techniques can work in a synergistic fashion to provide fundamental new insights in the process of viral adaptation. Hopefully such work will help stimulate more studies in this important emerging discipline.

Entomol. 41, 987-993.

6. Zárate, S. \& Novella, I. S. (2004) J. Virol. 78 , 12236-12242.

7. Truyen, U., Gruenberg, A., Chang, S. F., Obermaier, B., Veijalainen, P. \& Parrish, C. R. (1995) J. Virol. 69, 4702-4710. 